Musées, Patrimoine et Culture scientifiques et techniques

$126 \mid 2009$

novembre - décembre 2009

\title{
Une culture scientifique et technique au service d'une co-construction des savoirs
}

A scientific and technical culture serving a co-construction of knowledge

Jean-Pierre Cordier, Séverine Dessajan et Jacqueline Eidelman

\section{OpenEdition}

\section{Journals}

Édition électronique

URL : http://journals.openedition.org/ocim/209

DOI : 10.4000/ocim.209

ISSN : 2108-646X

Éditeur

OCIM

Édition imprimée

Date de publication : 1 novembre 2009

Pagination : 28-35

ISSN : 0994-1908

Référence électronique

Jean-Pierre Cordier, Séverine Dessajan et Jacqueline Eidelman, « Une culture scientifique et technique au service d'une co-construction des savoirs », La Lettre de I'OCIM [En ligne], 126 | 2009, mis en ligne le 01 novembre 2011, consulté le 19 avril 2019. URL : http://journals.openedition.org/ocim/209 ; DOI :

$10.4000 /$ ocim. 209 


\title{
Une culture scientifique et technique au service d'une co-construction des savoirs
}

\author{
Jean-Pierre Cordier, Séverine Dessajan et Jacqueline Eidelman *
}

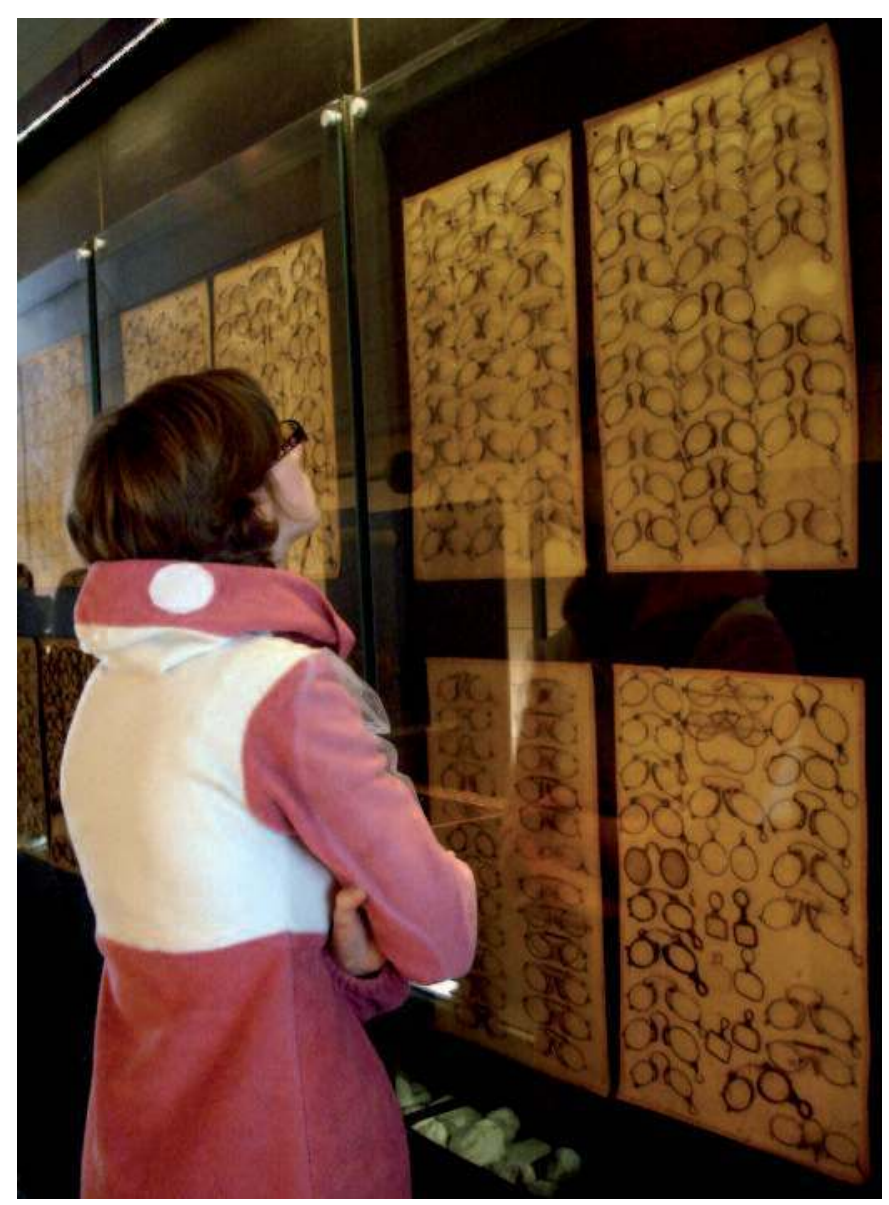

Musée de la Lunette à Morez (c) Fabien Lacaille

* Jean-Pierre Cordier, Séverine Dessajan et Jacqueline Eidelman sont chercheurs au CERLIS (Centre de Recherches sur les Liens sociaux), université Paris Descartes, UMR 8070-CNRS jacqueline.eidelman@culture.gouv.fr
Les musées des sciences et techniques et les centres de culture scientifique, technique et industrielle présentent des différences et des complémentarités dans la manière de concevoir et d'aborder le rapport entre la science et la culture : une analyse menée à partir de recherches consacrées à ce dispositif de diffusion de la culture scientifique et technique permet de constater une évolution de la demande sociale dans ce domaine et de mettre en lumière un ensemble de pratiques citoyennes participatives.

Depuis quelques décennies, une élévation générale du niveau d'instruction et une transformation des pratiques culturelles portent un nombre de plus en plus important d'individus à sonder l'opacité de la «boîte noire » des sciences et des technologies qui conditionnent largement les activités des sociétés contemporaines. Ainsi en même temps que les nouveaux moyens de communication ouvrent l'accès à un potentiel d'informations quasiment illimité, des initiatives plus ou moins institutionnalisées se développent pour répondre à la demande croissante de citoyens désireux de s'intégrer au périmètre des décisions qui dans ces domaines affectent leurs conditions et modes de vie. Cette quête, où sont mis en question les processus décisionnels classiques fondés sur un privilège d'expertise savante, génère une production symbolique qui se veut tout à la fois « contrepoint " aux savoirs légitimes et « contre-pouvoir » dans un contexte de mobilisation pour la cause publique. Non seulement, une telle posture est à l'œuvre dans le 
domaine Sciences et Société, mais ce domaine s'est même institué comme emblématique de son expérimentation sociale. Comment le dispositif formel de diffusion de la culture scientifique en France intègret-il de telles problématiques ? Nous tenterons de répondre en reprenant les apports d'un programme d'études et de recherche - que réalise le CERLIS (Centre de Recherches sur les Liens sociaux) en interaction avec la demande institutionnelle (1) - consacré à l'analyse de l'offre de Culture Scientifique Technique et Industrielle (CSTI) en France, son organisation, ses logiques, et sa fonction sociale.

\section{Les nouveaux modes d'élaboration et d'organisation des savoirs}

À la source du questionnement, se trouve l'observation de deux courants en sens contraire quant aux modes d'élaboration et d'organisation des savoirs qui régissent les processus de décision dans le domaine des actions collectives.

D'un côté, c'est le perfectionnement continu du mouvement de rationalisation de l'ensemble des activités sociales qui progresse en étendue et en profondeur, en masse et en vitesse, aux plans quantitatif et qualitatif, grâce aux puissantes capacités de traitement et de diffusion de l'information qu'apportent les nouvelles technologies. Parmi les effets produits, on observe la naissance de nouveaux instruments de gestion économique et de contrôle social, interfaces perfectionnées d'adéquation entre l'offre sociétale et une demande individuelle d'autant mieux régulée qu'elle est anticipée rationnellement. Pour ce faire, des systèmes d'expertise chargés de produire les connaissances spécialisées pour la conception, le développement et le transfert de ces instruments sur la place publique sont mis en place.

De l'autre côté, des initiatives se multiplient à propos de domaines très variés et chez des groupes sociaux hétérogènes qui ont en commun une démarche et une stratégie de ré-appropriation des processus de décisions à partir « d'alter-savoirs » susceptibles d'œuvrer comme des « contre-pouvoirs ». Ces autres savoirs revendiquent une fonction d'argumentaire critique susceptible de figurer aux côtés des connaissances produites dans le respect des normes de la légitimité scientifique et de l'efficacité organisationnelle, avec lesquelles ils interagissent au plan des représentations et des pratiques. Ils relèvent d'une expertise différente, celle d'acteurs en situation mus par la dynamique d'une prise de conscience collective. Ces démarches se réclament de pratiques citoyennes dans le sens où elles s'insurgent contre un défaut de démocratie et des logiques d'efficacité imposées de façon technocratique. Elles entendent questionner des logiques d'actions trop souvent présentées comme un échafaudage naturel de déterminations cohérentes et faire reconnaître le bien fondé de formes de coopération et de co-décision. Enfin, cette reconnaissance de savoirs dont la légitimité ne répond pas aux seuls critères académiques et dont la valeur heuristique relève de cette «philosophie du non » analysée par Bachelard, intervient à une période de l'histoire des idées marquée, dans les sociétés occidentales, par une montée en puissance de la revendication des individus à exister par et pour eux-mêmes, comme porteurs d'une intelligence du monde où ils vivent, en réaction à la tendance d'une homogénéisation des cultures à travers la mondialisation des échanges matériels et culturels.

Au niveau des pratiques sociales, la mise en place de procédures participatives multipartites peut notamment correspondre à une phase du fonctionnement d'un organisme caractérisée par une production intellectuelle d'acteurs de plusieurs horizons, où s'associent

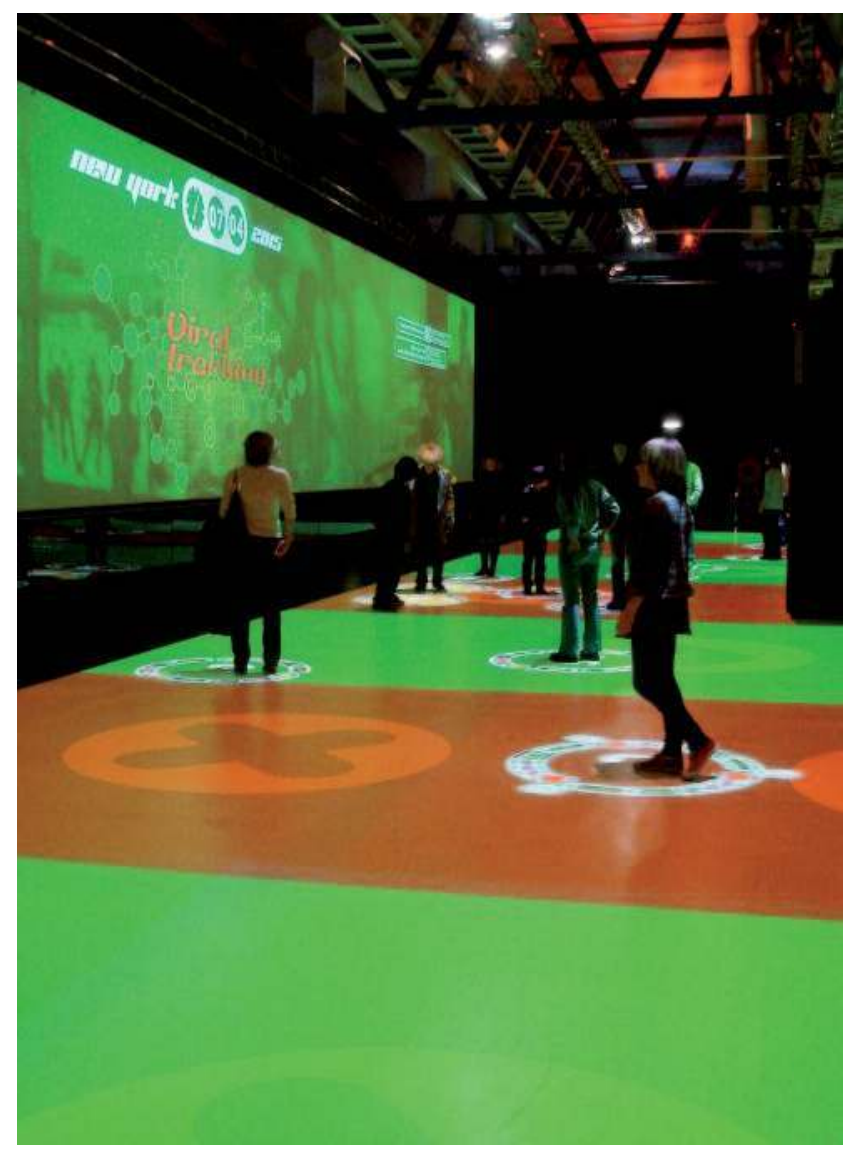

Jeu de simulation, dans l'exposition Épidemik à la Cité des Sciences et de l'Industrie (2009), dans lequel chaque participant peut agir sur le scénario d'une épidémie, en tant que citoyen ou décideur. ๑) Fabien Lacaille 
un état de connaissances, des représentations tirées d'une pratique ou d'autres sources, des présupposés idéologiques d'ancrages différents. À travers ce type d'expérience, c'est pour une institution d'accueil sa capacité de s'ouvrir à l'expression d'un public, considéré autrement qu'au titre de simple clientèle, qui est mise à l'épreuve, en même temps que sa capacité d'évoluer en tirant profit de tels apports. Et au niveau de l'individu, c'est tout l'apport qu'une prise de parole, au cours d'un débat contradictoire dans des conditions propices à s'affranchir d'une position de dépendance culturelle, peut lui procurer, avec la perspective, dans certaines occasions, d'être en situation de co-auteur d'une activité à finalité créatrice - le principal enjeu pour la construction de la personne étant la transférabilité de ces apports dans d'autres contextes et supports d'information (télévision, presse...) susceptibles d'un questionnement.

Pour prendre la mesure de ce phénomène on s'est alors interrogé, dans les cadres institutionnel et culturel qui offrent aux acteurs extérieurs des situations où ils peuvent s'impliquer, sur la façon dont ces derniers œuvrent, selon des formes et dans des conditions variées, à des mises en débat de savoirs reconnus ou émergents. Dans l'approche retenue, ces situations « participatives » ou « collaboratives » ont en commun de s’inscrire dans la problématique générale des pratiques de médiation considérées du point de vue de la réception, c'est-à-dire analysées « vues d'en bas », du point de vue de sujets situés au bout de la chaîne de transmission des savoirs. Mais également c'est sous les aspects d'une transaction réflexive que sont étudiées les modalités et les logiques interactives, pro- et rétro- actives.

\section{Deux institutions majeures du champ de la culture scientifique et technique sont concernées : les musées et les centres de culture scientifique technique et industrielle}

L’hétérogénéité du dispositif de CSTI français est le reflet d'une politique, plus ou moins régulière et plus ou moins harmonieuse, menée par le ministère de la Recherche depuis les Assises de la Culture scientifique et technique de 1982 et, depuis lors, relayée largement par les Régions à l'occasion des Contrats de Plan État-Région. Aussi bien, le nombre et le genre des structures (muséums, musées, centres de culture scientifique, technique et industrielle), le type et la forme des actions qu'elles mènent, les acteurs (État, collectivités territoriales, institutions publiques, associations, entreprises privées...) qui leur apportent leur concours en matière de fonctionnement, d'équipement et de personnel, n'ont cessé d'augmenter et se diversifier. Dans la phase 2004-2007, ce sont l'élaboration d'un référentiel commun d'évaluation de l'action des structures de diffusion, l'analyse qualitative des actions et ateliers à caractère scientifique en milieu scolaire, la cartographie de l'offre de CSTI en France qui ont polarisé l'attention. L'ensemble de ces résultats met en lumière une contribution non négligeable de ces structures à la médiation des connaissances scientifiques et au dialogue Sciences/Société. Les deux types d'institutions dont le rôle est prépondérant dans la diffusion des sciences et des techniques (les musées et les centres de culture scientifique technique et industrielle) forment des ensembles à la fois complémentaires et très différents dont les caractéristiques sont susceptibles d'influer sur la définition, la conception et la mise en œuvre des situations participatives que l'on propose d'étudier.

\section{L'institution muséale des sciences et des} techniques, un champ de pratiques en redéfinition La situation des musées est singulière à bien des égards, que ce soit sous l'angle de l'histoire et de l'organisation de ces institutions, des partis pris épistémologiques et des formes muséologiques ou de la sociologie des publics ${ }^{(2)}$.

Ainsi, pour situer brièvement les musées de sciences et de techniques, on retiendra qu'ils sont les héritiers d'une histoire séculaire ancrée dans une tradition humaniste de conservation et de diffusion des biens culturels; qu'ils sont une chambre d'écho à la

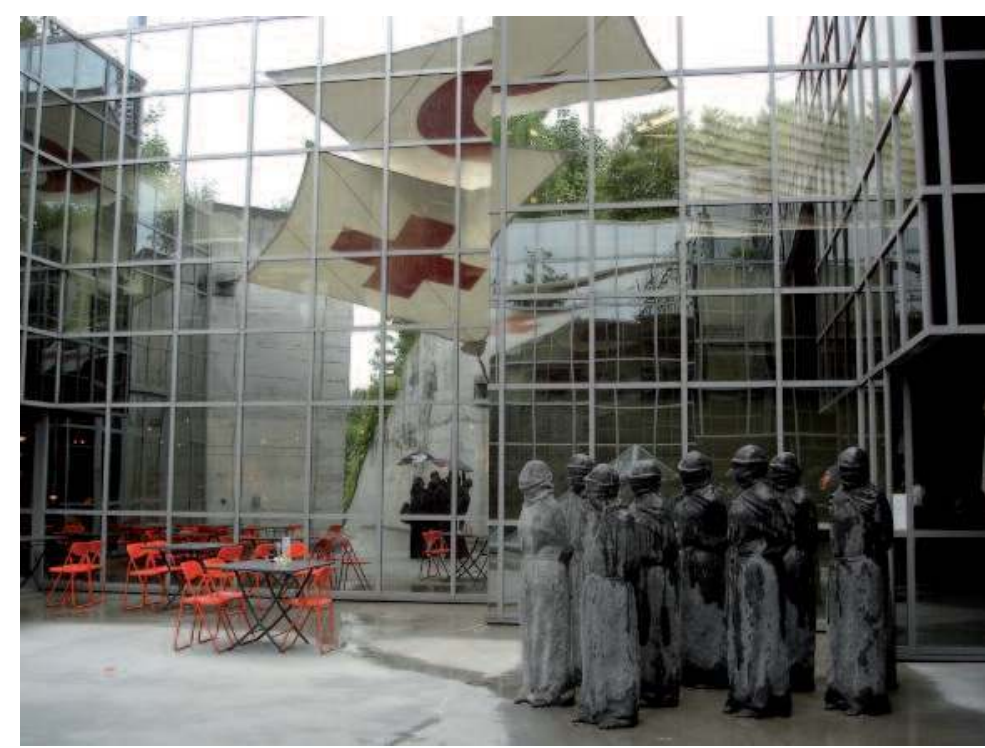

Entrée du musée de la Croix Rouge à Genève (c) Fabien Lacaille 
recherche, à son organisation, à ses logiques de production; qu'ils constituent des équipements à dimension régionale, nationale voire internationale dont la monumentalité est l'illustration; que généralistes ou spécialisés, ils traitent de thématiques qui couvrent l'éventail des connaissances, entre le savoir académique et l'actualité de recherches en cours ; que l'exercice de leur mission de diffusion relève d'une culture de la médiation sur site fondée sur le média particulier qu'est l'exposition; qu'ils s'adressent, en principe, à un vaste public à l'instar du public scolaire et du public familial ; que le principe didactique dominant est celui de l'interactivité et de la transversalité, mais que l'empreinte du cadre scolaire et de ses curricula demeure relativement structurante ; enfin, que cet ensemble donne lieu à des représentations et à des attitudes ambivalentes, dans la mesure où des changements structurels récents et une ouverture à des publics de plus en plus diversifiés ne suffisent pas toujours à supplanter limage du musée de sciences comme « cathédrale » du savoir, souffrant d'un déficit de proximité.

Les CCSTI, un champ de pratique institutionnelle en quête d'identité

Pour leur part, les CCSTI se caractérisent bien autrement : ce sont des structures de création récente (le dernier quart du XXe siècle), à dimension locale ou régionale ; ils sont essentiellement fréquentés par un public de proximité ; même s'ils sont en principe généralistes, leurs thématiques sont en grande partie influencées par les ressources, la demande et les potentialités de leur environnement - qu'il s'agisse du milieu naturel, du tissu industriel ou urbanistique, des soutiens institutionnels; leurs objectifs culturels font une large part à la mise à jour des représentations scientifiques et techniques, à l'actualité de la recherche et aux innovations technologiques, ainsi qu'aux débats sociétaux ; leur statut associatif et leurs modes de financement impliquent souvent de faire appel à des bénévoles ; ils ont recours à un éventail diversifié de médiations qui, selon les établissements, peuvent être sur site et/ou hors site dans une stratégie de rencontre d'un public éloigné ou empêché. Mais contrepartie de cette jeunesse et de son foisonnement, ils restent en butte à un déficit de légitimité dont témoigne leur évolution.

À l'origine de ce champ d'institutions médiatrices se trouve la mise en place, en 1995, d'un cadre de rencontre et de coopération, la "Réunion ", qui rassemblait des directeurs de centres dans un double objectif d'échange d'expériences et de savoir-faire dans l'exercice de leurs responsabilités institutionnelles et d'un rassemblement de forces pour la reconnaissance et le développement de leur action. Dans le fil de cette voie, la "Réunion des directeurs de CCSTI » s'est transformée en une « Réunion des CCSTI » dont les membres ne sont plus les responsables mais les structures - transformation qui s'est accompagnée de l'intégration des présidents et de la mise en place d'un conseil scientifique national, facteurs de stabilisation. Par ailleurs, pour parfaire le processus de légitimation, des contacts furent pris avec l'administration de tutelle (dépôt du nom « CCSTI » auprès de l'INPI) et de parlementaires pour aboutir à la rédaction conjointe par des membres du ministère de la Recherche et le président de l'association la « Réunion des CCSTI » de la charte qui détermine « les droits et les devoirs de chacun ». Ce document, signé par le ministre Roger-Gérard Schwartzenberg le 27 avril 2001 engageait les deux parties : les centres de culture scientifique et technique adhérents de la Réunion, sur des missions, des objectifs et des fonctions ; l'État, sur la reconnaissance et le soutien (notamment financier) apportés aux centres.

À partir de cette étape, en vue d'apprécier les effets de la contractualisation, une étude fut confiée au CERLIS qui prit la forme d'une enquête fondée sur des monographies de centres et l'organisation de tables rondes inter-régionales. Elle a permis de prendre la mesure, in situ, du fonctionnement du réseau spécifique des CCSTI.

Dans un second temps, cet état des lieux a servi de base à une réflexion sur les conditions de faisabilité d'une grille d'indicateurs pour l'évaluation de l'activité

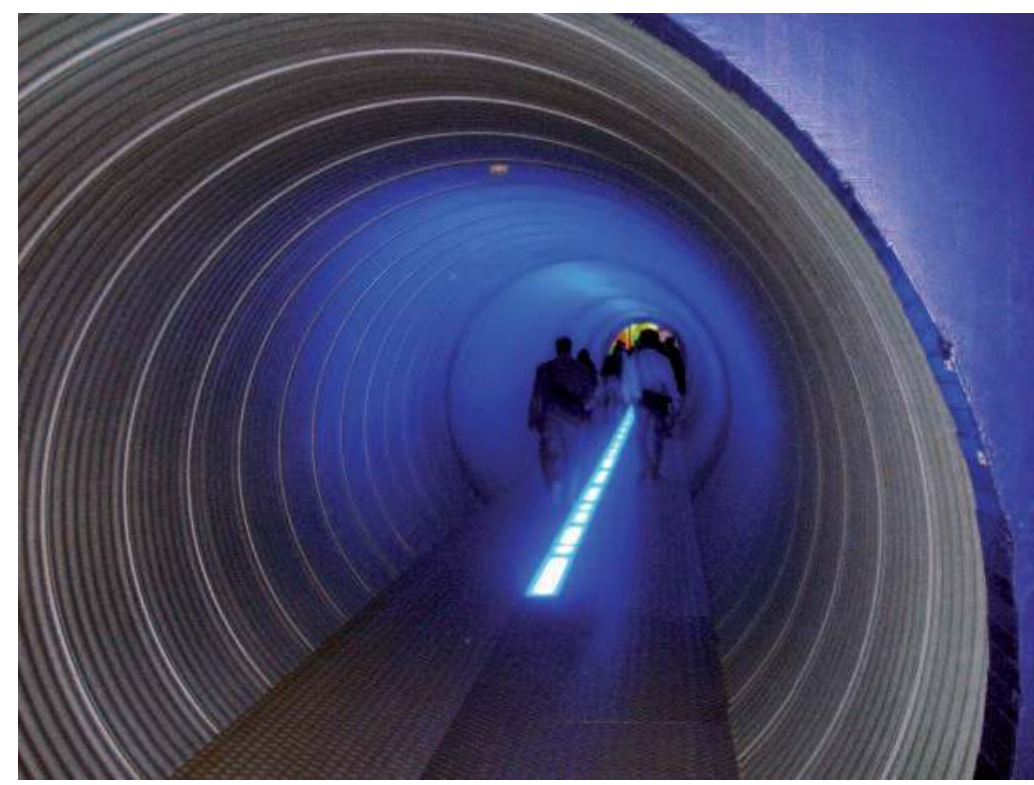

Exposition Portrait consacrée à l'identité et à l'image au Parc d'aventures scientifiques de Frameries (Belgique). (c) Catherine Ruppli 
de ces structures en termes d'organisation, d'impact et d'ancrage ${ }^{(3)}$. En effet plusieurs lignes de tension à retenir apparaissent au sein de ce réseau d'institutions à la fois en cours de professionnalisation et aux prises avec la nouvelle configuration des relations État/Collectivités territoriales dans le cadre de la régionalisation : entre communauté d'objectifs et de missions au plan national et manières variées de les mettre en œuvre en fonction du contexte local ; entre besoin de consolidation des structures et maintien d'une souplesse des modes d'action; entre bonne visibilité des actions pour s'assurer une position phare et une activité de service assez discrète pour valoriser les partenaires. Par ailleurs si l'évaluation trouve sa justification au plan éthique et est perçue comme un mode de régulation des logiques d'action, elle doit être aussi capable de traduire les contraintes de l'action concertée (différents niveaux et partenariats) ; et de même, être lié par la Charte ne signifie pas forcément que chaque CCSTI doit assumer l'ensemble des actions qui correspond au réseau, d'autant que la taille et les moyens des structures sont suffisamment inégaux et les contextes hétérogènes pour que se pose la question des économies d'échelle et de la mutualisation des ressources.

Après avoir pris acte de ces données, au cours des années 2006-2007, le ministère et la Réunion des CCSTI ont procédé à une réflexion sur la labellisation des CCSTI à travers la création d'un Comité de pilotage et la mise au point d'un protocole de labellisation. À l'automne 2007, ce protocole a été envoyé aux 34 CCSTI membres de la Réunion et entre décembre 2007 et juin 2008, le Comité s'est réuni pour analyser les candidatures et réfléchir à la labellisation d'autres structures ${ }^{(4)}$.

\section{Les divers modes de prise en compte des formes de coopération et de participation citoyenne dans la construction des savoirs}

L'institution muséale des sciences

et des techniques : un laboratoire d'expérimentation décisionnelle

Dans la suite des travaux réalisés depuis plus de vingt ans par certains membres du CERLIS, une nouvelle série a été inaugurée récemment qui participe au questionnement posé par l'évolution sociale des processus décisionnels : elle concerne la mise en place, l'observation et l'analyse des premières expériences de muséologie participative introduites en France, sous la forme de comités de visiteurs. D’abord au musée de l'Homme (2005-2006) ${ }^{(5)}$, puis à la Cité des Sciences et de l'Industrie (2007-2009), et, dans les mois qui viennent, au musée des Confluences à Lyon dont l'inauguration est prévue à l'horizon $2011^{(6)}$.

Ces comités de visiteurs représentent une nouvelle instance «muséale ». Tels qu'ils seront étudiés dans le cadre du programme de recherche, ils constituent un dispositif inédit de (re)fondation du musée par l'implication de ses publics. En tant qu'objet de recherche, ce type de concertation constitue une phase nouvelle à la fois complémentaire et originale des analyses menées au CERLIS sur les publics de la culture scientifique qui peut être considérée comme emblématique des nouveaux modes de participation dans le cadre des musées. Après avoir acclimaté en France la problématique de l'évaluation ${ }^{(7)}$ et en particulier celle de l'évaluation préalable et formative, l'équipe du CERLIS pousse jusqu'à son terme la problématique de la réception avec l'analyse, en situation, de l'horizon d'attente. Au lieu de prendre en compte les réactions des visiteurs d'une exposition de préfiguration pour améliorer la réception d'une muséographie déjà conçue, la démarche des comités de visiteurs se situe plus en amont, au moment où la thématique même d'une exposition est encore l'occasion d'un débat. Concrètement, elle prend la forme d'un groupe d'une douzaine de visiteursusagers différenciés quant au statut social (genre, âge, milieu), à leur rapport au musée et à leur pratique des expositions, lesquels se réunissent tous les mois pendant une année renouvelable en compagnie de concepteurs d'expositions, représentants du musée, et de chercheurs en situation d'observation-animation. Au

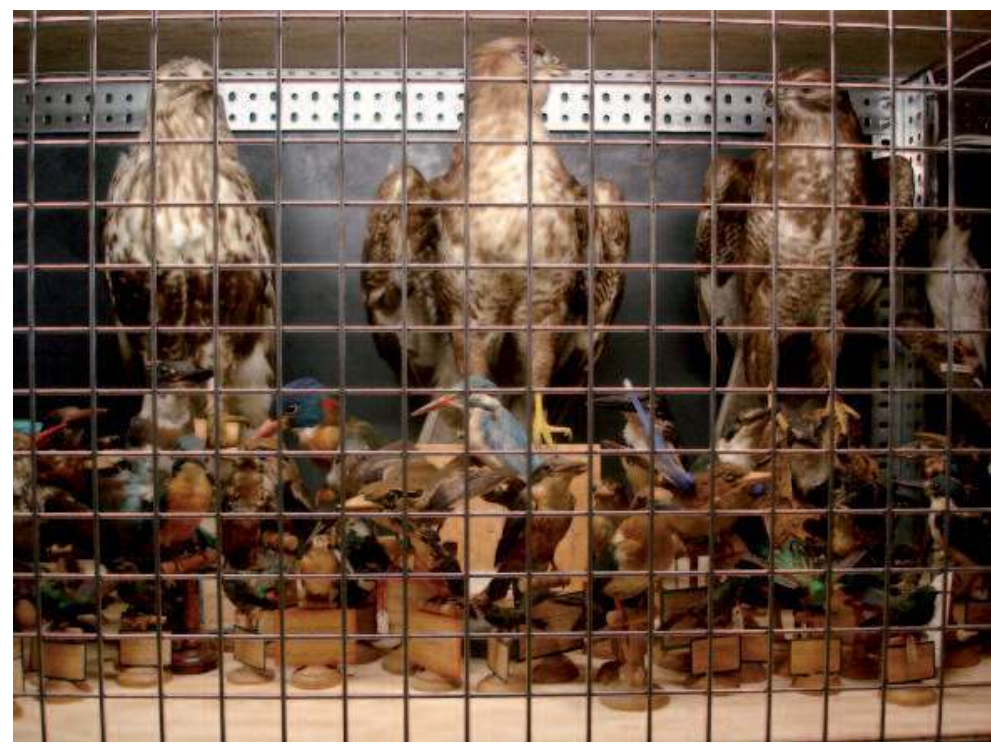

Détail d'une exposition sur les collections des muséums d'Histoire naturelle, présentée au musée zoologique de Zurich en 2008. (c) Fabien Lacaille 
fil des séances, les projets conceptuels et pratiques de la muséographie, définis en termes d'objectifs et d'attentes vis-à-vis de la réception du public, sont exposés et discutés en commun. Si elle enrichit la compétence du visiteur-usager et illustre jusqu'à quel point le musée est un médiateur du social et de l'individu, la démarche impose, aussi, au musée, une transaction réflexive avec ses publics. Cette nouvelle instance paraît, en effet, essentielle si l'on souhaite réellement modifier en profondeur les relations entre les publics et les musées. À mieux connaître ses publics, à être prêt à s'entendre avec eux, à leur reconnaître une expertise propre, n'est-on pas mieux armé pour faire du musée un espace public, c'est-à-dire, un espace de délibération et de réinvention de la culture ?

\section{Les CCSTI, un champ d'exploration} et $d^{\prime}$ interrogation identitaires

Jusqu’à présent, les structures de la « Réunion des CCSTI » engagées dans le processus de reconnaissance de leur action ont fait essentiellement l'objet d'évaluations institutionnelles sur leurs conditions et mode de fonctionnement et n'ont presque jamais été le sujet d'études de réception auprès de leurs publics. Dans le premier de ces champs d'intervention, les travaux de recherche ou d'expertise missionnés à l'équipe du CERLIS par des instances à vocation nationale (la Direction puis la Mission de l'information et de la culture scientifique technique et industrielle du ministère de la Recherche) ou internationale (Institut de Recherche pour le Développement pour le compte de la Direction générale de la Coopération internationale et du Développement du ministère des Affaires Étrangères) ont permis d'établir un état des lieux de la culture scientifique et technique, que ce soit pour la France ou dans des pays d'Afrique associés à la Zone de solidarité prioritaire. Leurs résultats constituent la base d'une connaissance et d'une intelligence du milieu des CCSTI susceptible de fournir à l'avenir le cadre d'études de réception centrées sur les publics ${ }^{(8)}$.

Pour l'heure, il ressort de cet inventaire analytique des caractéristiques organisationnelles et opérationnelles de l'ensemble des CCSTI que pour l'essentiel, ces jeunes structures, totalement engagées dans la double voie de l'institutionnalisation et de la professionnalisation, accèdent à une nouvelle étape de développement qui renforce leur rôle de médiation, que ce soit au niveau du type de public ou par des formes d'interactions qui s'inspirent de la problématique du débat citoyen. Certains des indicateurs retenus comme critères de leur labellisation témoignent de cette orientation :
- La fonction d'organisation. À proximité des acteurs locaux de culture scientifique et technique dont ils sont souvent partenaires, les CCSTI ont mission d'assumer un rôle de tête de réseau local voire régional, mobilisateur et fédérateur d'initiatives. D'où la question qui leur est posée de savoir si et comment cette présence forte sur le terrain s'accompagne d'une sensibilité particulière à l'évolution de la demande sociale en termes de culture scientifique et technique.

- Les stratégies de communication. Elles sont multiples. Être un lieu sans murs ou avoir un espace ouvert aux publics correspond à deux types de projets distincts : se consacrer entièrement à une fonction réticulaire ou incarner un nouveau type d'équipement culturel. On observe également que la part des échanges virtuels, grâce aux nouvelles technologies d'information et de communication et les moyens offerts par Internet s'est surdéveloppée ces dernières années. Enfin certains CCSTI apparaissent à la pointe de l'innovation tant dans le contenu que dans la

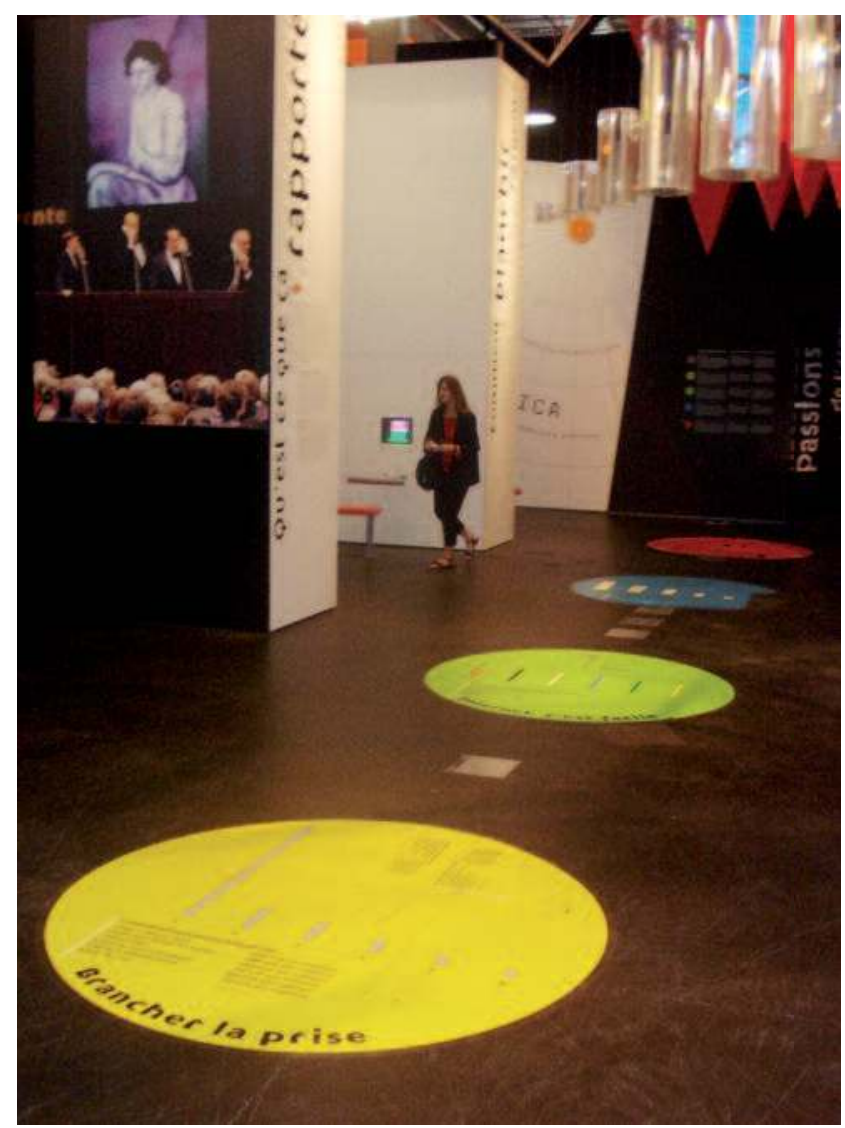

Exposition L'argent au Parc d'aventures scientifiques de Frameries (Belgique) (C) Catherine Ruppli 
forme de leurs outils de médiation pour toucher le public le plus large, tant les jeunes qu'un public non familier des sciences et techniques. Dès lors, comment cet ensemble très divers d'initiatives s'inscrit-il par rapport aux changements en cours dans le rapport avec la science et la technique d'un côté, et la société de l'autre, tel que le vit actuellement une partie du public ? Quels sont les apports respectifs de ces différentes démarches?

- Le lien entre recherche et développement et la professionnalisation de sa médiation. La collaboration des CCSTI avec des chercheurs, des industriels, des ingénieurs techniciens montre combien l'articulation entre recherche fondamentale, recherche appliquée et recherche développement est au centre des contenus de leurs activités - même si les deux dernières sont davantage mises en avant sous la forme de productions matérielles (expositions, ateliers...) et immatérielles (conférences, bar des sciences...) qui font appel de plus en plus à des professionnels de la médiation culturelle et à un renouvellement du répertoire des compétences. Quels sont les conditions et les effets de cette recomposition des fonctions?

Pour conclure, entre les deux principales structures institutionnelles, les musées et les CCSTI, qui font øuvre de médiation dans le domaine des sciences et

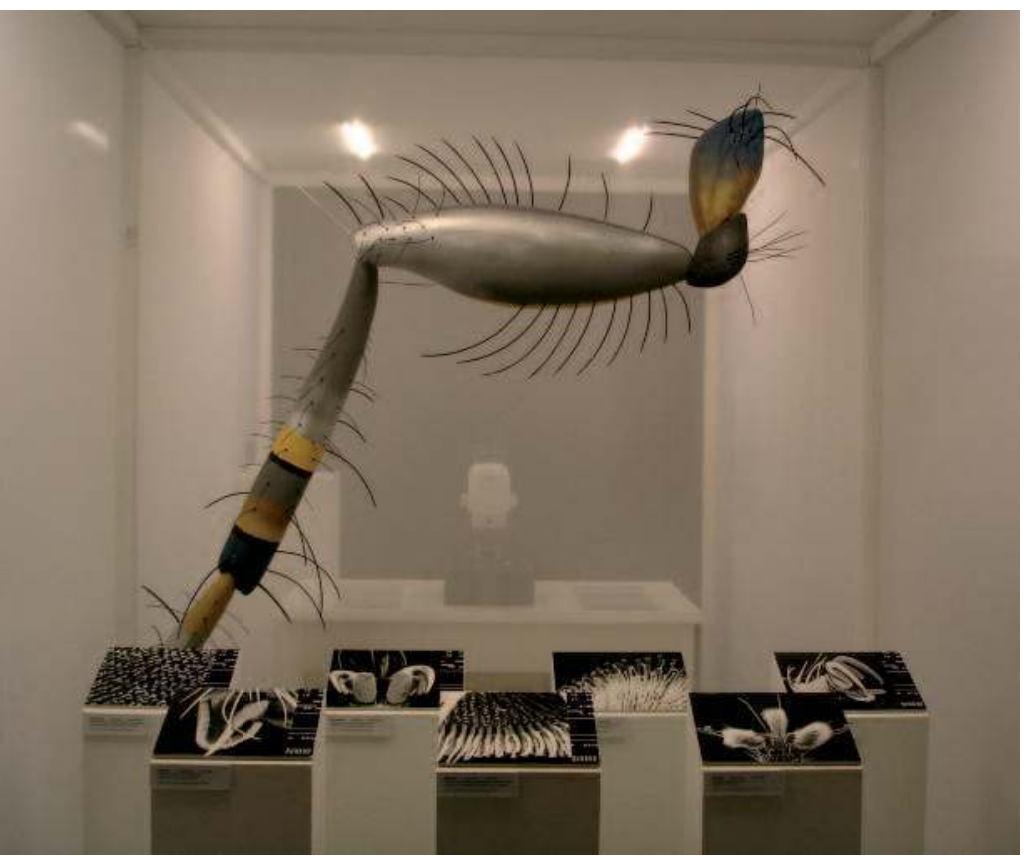

Exposition Mouche, créée par le muséum d'Histoire naturelle de Neuchâtel et présentée en 2007 au Muséum national d'Histoire naturelle à Paris en 2007 (c) Fabien Lacaille des techniques, des philosophies d'action jusqu'à présent différentes justifient une investigation en perspective, fondée sur des comparaisons heuristiques... Soit, par exemple, la nature du public et ses attentes supposées en terme de thématique : celui des musées serait-il plus «savant", d'un niveau scientifique et d'une exigence supérieurs parce qu'on y viendrait s'instruire sur la « vraie » science ou les dernières « curiosités intellectuelles»? Tandis que celui des CCSTI serait plus «populaire", éventuellement plus « militant », c'est-à-dire plus curieux de s'informer sur des sujets d'actualité mal connus ou controversés pour s'en faire une opinion? Autre exemple, sur la façon dont est abordée une même thématique : selon qu'il s'agisse d'un musée ou d'un CCSTI y a-t-il des spécificités, liées à des traditions et expériences différentes, dans le mode d'approche et le propos, en particulier dans la façon de concevoir et négocier le rapport entre la science et la culture ? Dans quelle mesure et comment l'un ou l'autre champ institutionnel prend-il en compte les changements dans la société qui concernent à la fois la détermination des cadres de vie et le rôle revendiqué par les personnes qu'ils affectent? Suscitent-ils les mêmes rapports à la science, à la culture scientifique?

Tout comme la question des mises en œuvre variées d'une problématique émergente, se pose celle de la co-construction des savoirs. En effet, une interrogation concerne en particulier les situations participatives de co-construction de savoirs et de culture. Procédures ou interfaces particulières, elles peuvent se présenter sous différents formats : interaction verbale, activité matérielle, groupe de concertation, jeu virtuel.... Qu'est-ce qui constitue l'originalité et l'apport spécifique de chaque dispositif ? Quelles en sont les limites? Dans quelle mesure le produit de leur intervention est-il transférable dans d'autres domaines, d'autres contextes... ? Dans ces deux types de structures, différentes opérations déclinent la question de la co-construction de projets culturels. En conséquence, de telles analyses ont pour but de tester l'idée selon laquelle des dispositifs de médiation spécifiques sont susceptibles de catalyser une forme de partenariat qui place le public en position, tierce ou contradictoire, d'un émetteur capable d'influer sur le discours ou l'événement auquel il lui est donné de participer.

Enfin, il paraît important de rappeler que l'articulation des deux thématiques abordées dans cet article : celle de la «co-production» des sciences, des individus et du social, avec celle des pratiques de la 
« culture scientifique et technique », montre qu'elles participent à la construction de l'identité de la personne, dans une perspective de responsabilité citoyenne. Ainsi, les contextes de co-construction de projets culturels dans les domaines «sciences et société » sont aussi des nouvelles manières de mise en culture tout en permettant l'émergence d'un nouveau mode de fabrique de l'individu contemporain.

\section{Notes}

(1) Une partie des travaux que l'on évoquera entre dans le cadre de la convention de recherche pluriannuelle entre la Mission de l'information et de la culture scientifiques et techniques et des musées (ministère de l'Enseignement supérieur et de la Recherche), l'université Paris Descartes et le CNRS. Une autre partie est issue de conventions de coopération signées respectivement avec la Cité des Sciences et de l'Industrie et le Muséum national d'Histoire naturelle.

(2) Le Marec, J. (dir.) Évolution des rapports entre sciences et société au musée, Culture et Musées, n¹0, 2007 ; Eidelman, J. et Laffon, F. Des publics pour une refondation du musée de l'Homme, in Mohen, J.-P. (éd.), Le nouveau musée de l'Homme. Paris : Odile Jacob, 2004, pp. 223 251 ; Eidelman, J. (coord.) Qui visite les musées de sciences ?, la Lettre de l'OCIM, n55, janvier-février 1998.

(3) Bergeron, A., Eidelman, J., Saurier, D., Dessajan, S. et Cordier, J.-P. Évaluation des contextes et modalités de diffusion de la culture scientifique et technique. Enquête qualitative. Tome 1 : État des lieux et fonctionnement du réseau des CCSTI. Tome 2 : Corpus de monographie. Paris :
Mission de la culture et de l'information scientifiques et techniques et des musées, CERLIS, 2004, 203 p. et 244 p.

(4) Eidelman, J. (dir) et Dessajan, S. Évaluation des contextes et modalités de diffusion de la culture scientifique et technique, Analyse des protocoles de labellisation. Paris : Bureau de la culture et du patrimoine scientifiques et techniques de la Mission de l'information et de la culture scientifiques et techniques et des musées (ministère de l'Enseignement supérieur et de la Recherche), décembre 2008, 40 p.

(5) Dessajan, S. Une expérience innovante au musée de l'Homme : un comité de visiteurs ou comment les usagers du musée prennent la parole. Paris : La Documentation Française, Collection Musées-Mondes, n¹.

(6) Eidelman, J. et Dessajan, S. La muséologie participative et l'émergence d'une nouvelle instance au sein des musées. Bilan des séances de Comités de visiteurs à la Cité des Sciences et de l'Industrie. Rapport de recherche, décembre 2008 (chercheurs associés : De Mengin, A. Cité des Sciences et de l'Industrie).

(7) Eidelman, J. et Van Praët, M. (dir.) La muséologie des sciences et ses publics. Paris : PUF, 2000, $352 \mathrm{p}$.

(8) Dessajan, S. et Ramos, E. La culture scientifique et technique dans dix pays de la zone de solidarité prioritaire. Tome 1 (2006): État des lieux. Tome 2 (2006) : La culture scientifique et technique au Maroc, Tome 3 (2007) : La Culture scientifique au Burkina Faso, Tome 4 (2008) : La Culture scientifique à Madagascar, en quête d'acteurs et d'activités, Paris, Institut de Recherche pour le Développement, 146 p., 96 p., 85 p. et 62 p. 